\title{
A discrete-time formulation for the variable wave speed scattering problem in two dimensions
}

\author{
Jeffrey L Frolik and Andrew E Yagle \\ Department of Electrical Engineering and Computer Science, The University of Michigan, \\ Ann Arbor, MI 48109-2122, USA \\ Received 24 April 1995, in final form 4 March 1996
}

\begin{abstract}
Motivated by electromagnetic wave propagation in media where permittivity varies in two dimensions, we address the problem of wave scattering for two-dimensional (2D) media having variable speed. Wave speed variations are shown to produce scattering which can be represented in terms of a Schrödinger scattering potential. The wave equation problem is thus reformulated as a Schrödinger equation inverse potential problem, with a variable wave speed. Throughout it is assumed that wave speed varies smoothly and slowly such that a finite-difference approximation is valid, defining a discrete inverse scattering problem. For this discrete problem, we define an equivalent medium on a variable-mesh grid for which the wave speed is constant throughout, yet the equivalent medium has the same scattering response as the actual variable wave speed medium. Going from actual to equivalent medium entails spatially warping the medium, while going from equivalent to actual entails spatial dewarping. The discrete-time forward and inverse scattering problems are then formulated and solved using the equivalent medium. A numerical example illustrating the introduced concepts is presented.
\end{abstract}

\section{Introduction}

Inhomogeneous media are characterized by parameters which vary spatially. Waves propagating through such media are affected by these parameter changes. In this paper we investigate the wave scattering in 2D media in $x z$-space for which the wave speed $c$ is a function of position $\{c(x, z)\}$ and is slowly and smoothly varying. For brevity we will refer to the vertical direction ( $z$ coordinate) as depth and to the horizontal direction ( $x$ coordinate) as breadth.

Our motivation for considering this problem is that fast layer stripping algorithms can be easily implemented for the constant wave speed problem, but for the variable wave speed problem implementation is presently difficult in the 1D case and impossible for higher dimensions. Our goal is to show that the scattering problem for variable wave speed media can be solved using equivalent constant wave speed warped media. The scattering of the warped medium is identical to that of the actual medium in time, but in space the reconstructed warped medium has the right effective index values at the wrong places. Since our warping and dewarping process is dependent only on the effective index values of the actual and equivalent media, respectively, one can be found easily from the other.

Our approach differs threefold from other numerical methods such as finite-difference time domain (FDTD) [3] and transmission line matrix (TLM) methods [6]. First, FDTD and TLM methods are recursive methods for the forward problem only. Our approach implements fast algorithms for both the forward and inverse problems. Second, FDTD 
and TLM methods are based on discretized Maxwell curl equations. We develop a new finite-difference approximation of the Schrödinger equation applicable to variable wave speed media. Finally, FDTD and TLM discretizations use a uniform spatial mesh, whereas our approach uses a conformal variable spatial mesh which is equivalent to uniformly discretizing the actual medium temporally. In addition, we approximate a continuous medium as piecewise constant over a discrete time interval versus piecewise constant over a discrete spatial interval. We assume the wavelength of the propagating wave is much longer than the discretization interval so that surface roughness can be ignored. Recent work in FDTD has considered a non-uniform spatial mesh for determining fields around small features; however the lattice is still rectangular and is boundary driven, not medium driven [3].

Our discrete Schrödinger equation for the variable wave speed problem (28) can be viewed as a variable-mesh finite-difference approximation. Our work to date has started off with such discrete equations as the formulation of the actual problem to be solved $[1,2,7]$. In this previous work the discrete equations have clear finite-difference interpretations and therefore applications. In this paper, we are solving a discretized problem, which is an approximation to another continuous problem.

Specifically the contributions of this paper are fourfold. First, the concept of an equivalent constant wave speed medium having identical scattering characteristics to the actual variable wave speed medium is introduced. Second, methodology for finding the equivalent medium from the actual, or vice versa, consisting of spatially warping the medium is developed. Third, a new variable-mesh finite-difference approximation of the Schrödinger equation applicable to variable wave speed media was developed. Finally, new discretetime 2D formulations of the forward and inverse problem for variable wave speed media which use the interim equivalent warped medium are presented. With these equivalent representations and the results discussed in [1], layer stripping algorithms can be used on a variable speed medium. The important point to note is that when layer stripping algorithms are applied to scattering data from a variable wave speed medium it is the warped constant speed medium that is being reconstructed. By understanding the relationship between the actual and warped media the former can be found from the latter.

This paper is organized as follows. In section 2, we recast the general wave equation in terms of travel time and energy normalized field components to obtain the Schrödinger equations. In section 3, the concept of the equivalent warped medium is introduced. The equivalent warped medium has the same scattering response as the actual variable wave speed medium, but has uniform wave speed. We develop warping methodology for 1D media using an intuitive example and extend these results to the 2D case. In section 4, we begin with the most general discretization of the Schrödinger equation using the central difference approximation. In the subsequent subsections, variable-mesh finite-difference approximations are used to find a constant wave speed Schrödinger equation equivalent to the variable wave speed Schrödinger equation. Layer stripping algorithms for the forward and inverse problem developed in [1] are quickly reviewed in section 5 and section 6, respectively. To illustrate the introduced methodology, we present in section 7 a numerical example of scattering from a 2D inhomogeneous wave speed media. Section 8 concludes with a summary and suggestions for future research.

\section{The Schrödinger equation for $2 \mathrm{D}$ variable wave speed media}

The main results of this paper are obtained by discretizing the variable-velocity Schrödinger equation using a variable-mesh grid to incorporate the effects of varying wave speed. In this 
section, we present the continuous analogues of our discrete results. In doing so, we illustrate how some second-order effects are neglected in finite-difference approximations and thus do not appear subsequently. Nonetheless, the discrete results are valid if the discretization lengths are sufficiently small to make the finite-difference approximation (which must be used in any case) valid. In this paper, we start with finite differences, rather than end with them.

We begin by recasting the general wave equation in terms of travel time and energy normalized field components. Using this formulation allows us to cancel out first-order partial derivative terms due to the spatial variation of wave speed. The result of this reformulation is the Schrödinger equation. Motivated by the problem of electromagnetic wave propagation in inhomogeneous dielectrics, we begin with Maxwell's curl equations

$$
\begin{aligned}
\nabla \times \boldsymbol{E} & =-\mu \frac{\partial \boldsymbol{H}}{\partial t} \\
\nabla \times \boldsymbol{H} & =\varepsilon \frac{\partial \boldsymbol{E}}{\partial t}
\end{aligned}
$$

where $\boldsymbol{E}=\boldsymbol{E}(x, z, t)$ and $\boldsymbol{H}=\boldsymbol{H}(x, z, t)$ are the electric and magnetic fields, respectively, and $\varepsilon$ and $\mu$ are the permittivity and permeability of the medium, respectively. Assuming a non-magnetic medium, $\mu$ will be constant $\left(\mu(x, z)=\mu_{0}\right)$. The electromagnetic wave equation for the electric field (2.2) is obtained by taking the curl of (2.1a) and substituting in $(2.1 b)$ to give

$$
\left(\nabla^{2}-\mu_{0} \varepsilon \frac{\partial^{2}}{\partial t^{2}}\right) \boldsymbol{E}=0 .
$$

Noting that $c(x, z)=1 / \sqrt{\mu_{0} \varepsilon(x, z)}$ is the wave speed at spatial coordinate $(x, z)$, we see that variable permittivity is equivalent to variable wave speed. For our purposes, we consider the scalar wave equation (2.3) noting that (2.2) is its electromagnetic form

$$
\nabla^{2} \psi-\frac{1}{c^{2}} \frac{\partial^{2} \psi}{\partial t^{2}}=0
$$

\subsection{ID case}

For ease of notation, we derive the 1D Schrödinger equation in this section and extend the result to $2 \mathrm{D}$ in the following section. For the $1 \mathrm{D}$ case, (2.3) becomes

$$
\frac{\partial^{2} \psi(z, t)}{\partial z^{2}}-\frac{1}{c(z)^{2}} \frac{\partial^{2} \psi(z, t)}{\partial t^{2}}=0 .
$$

Defining the energy-normalized wave field $\Psi=\psi / \sqrt{c}$ and changing variables from depth $\{z\}$ to travel time $\{\tau\}$ using

$$
\tau(z)=\int_{0}^{z} \frac{1}{c\left(z^{\prime}\right)} \mathrm{d} z^{\prime} \leftrightarrow \frac{\mathrm{d} \tau}{\mathrm{d} z}=\frac{1}{c}
$$

yields the well known Schrödinger equation in the time domain:

$$
\frac{\partial^{2} \Psi}{\partial \tau^{2}}-\frac{\partial^{2} \Psi}{\partial t^{2}}=\sqrt{c}\left(\frac{\partial^{2}}{\partial \tau^{2}} \frac{1}{\sqrt{c}}\right) \Psi .
$$

The scattering potential in (2.6) is given by $V=\sqrt{c}\left(\partial^{2}(1 / \sqrt{c}) / \partial \tau^{2}\right)$ which can be defined in terms of a reflectivity $r$

$$
V=r^{2}-\frac{\partial r}{\partial \tau}
$$




$$
r=\sqrt{c}\left(\frac{\partial}{\partial \tau} \frac{1}{\sqrt{c}}\right) .
$$

\section{2. $2 D$ case}

We extend the 1D derivation of the previous section by first defining two different spatial warping parameters: one as a function of depth $\left\{\tau_{z}\right\}$ and one as a function of breadth $\left\{\tau_{x}\right\}$ (compare with the single travel time variable $\tau$ used in the 1D case (2.5)):

$$
\begin{aligned}
& \tau_{z}(z, x)=\int_{0}^{z} \frac{1}{c\left(z^{\prime}, x\right)} \mathrm{d} z^{\prime} \leftrightarrow \frac{\partial \tau_{z}}{\partial z}=\frac{1}{c} \\
& \tau_{x}(z, x)=\int_{0}^{x} \frac{1}{c\left(z, x^{\prime}\right)} \mathrm{d} x^{\prime} \leftrightarrow \frac{\partial \tau_{x}}{\partial x}=\frac{1}{c} .
\end{aligned}
$$

Equations (2.8a) and (2.8b) are just $z$ and $x$ transformations, respectively, by the local wave speed $c(z, x)$. Note that the transformations given in (2.8) have a second-order dependency on each other. Assuming that the wave speed variations are locally isotropic, this dependency will thus not appear in the finite-difference approximations of section 4, and we therefore neglect it in the following. Hence, the spatial warping can be performed in either order (e.g., $x$ then $z$ or $z$ then $x$ ).

Beginning with the general wave equation (2.3) and proceeding as in the 1D case using the 2D spatial warping transformations given in (2.8), we obtain the 2D Schrödinger equation in the time domain,

$$
\frac{\partial^{2} \Psi}{\partial \tau_{z}^{2}}+\frac{\partial^{2} \Psi}{\partial \tau_{x}^{2}}-\frac{\partial^{2} \Psi}{\partial t^{2}}=V \Psi
$$

where the 2D scattering potential $\{V\}$ is now described in terms of the local reflectivities $\left\{r_{z}\right.$ and $\left.r_{x}\right\}$ (compare with their discrete 2D versions (4.3) and their continuous 1D version $(2.7 b))$

$$
\begin{aligned}
& V=\sqrt{c}\left(\frac{\partial^{2}}{\partial \tau_{z}^{2}} \frac{1}{\sqrt{c}}\right)+\sqrt{c}\left(\frac{\partial^{2}}{\partial \tau_{x}^{2}} \frac{1}{\sqrt{c}}\right)=\sqrt{c}\left(\nabla_{\tau}^{2} \frac{1}{\sqrt{c}}\right)=r_{z}^{2}+r_{x}^{2}-\frac{\partial r_{z}}{\partial \tau_{z}}-\frac{\partial r_{x}}{\partial \tau_{x}} \\
& r_{z}=\sqrt{c}\left(\frac{\partial}{\partial \tau_{z}} \frac{1}{\sqrt{c}}\right) \\
& r_{x}=\sqrt{c}\left(\frac{\partial}{\partial \tau_{x}} \frac{1}{\sqrt{c}}\right) .
\end{aligned}
$$

\section{Formulating the equivalent constant wave speed medium}

Consider an actual 1D lossless medium consisting of a stack of layers in the $z$ direction, each having the same length $\Delta=1$ (figure $1(a)$ ). We assume that the wave speed in each layer is quantized to a rational number (this is inevitable in a finite-precision computing environment anyway). This is equivalent to assuming that the slowness (reciprocal wave speed) in each layer is rational. Let $c_{0}$ be the least common denominator of the rational slownesses. Then the slowness in layer $i$ can be written as $N(i) / c_{0}$ for some integer $N(i)$ which can be different in each layer. This means that the wave speed in layer $i$ is $c_{0} / N(i)$.

The scattering of the actual medium is identical in time to the scattering of a medium constant wave speed $c_{0}$, but with layers having thickness $N(i) \Delta$ (figure $1(b)$ ). That is, slower wave speed layers are equivalent to thicker layers in the constant wave speed medium, as was shown in the numerical example of [2]. Since the medium we are considering is 
lossless, scattering magnitude effects are a function of the interface discontinuities only, not of layer thickness (there is no attenuation with distance). Indeed, there is no way of distinguishing between the responses of the actual and warped media. Note that interfaces of the actual and warped media are identical in magnitude and occur at the identical places in time, but different places in space. This is the basic idea behind a Goupillaud (equal travel time in each layer) layered medium, an assumption that was used extensively in the seismic exploration literature in the 1960s.

(a)

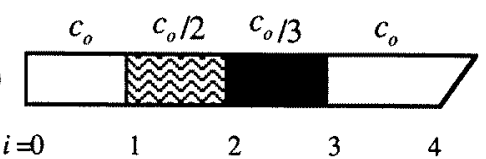

(b)

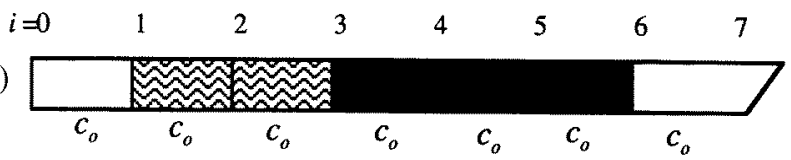

Figure 1. Actual and warped 1D medium example. (a) Variable wave speed (actual) medium; (b) constant wave speed (warped) medium.

The term warped medium is used because, as described above, we are warping the spatial dimensions of the actual medium, since the warped medium has constant wave speed and therefore the spatial and temporal discretizations are uniform throughout the medium (see section 4).

The warped medium can be reconstructed from the measured reflection response using layer stripping algorithms (e.g., Levinson and/or Schur algorithms [8]). The equivalent media cannot be distinguished without a priori knowledge that the reflections are caused not by density $(\rho(i)$ or $\mu(i))$ variations, but by wave speed variations $\left(c(i) \equiv(\mu(i) \varepsilon(i))^{-1 / 2}\right)$. For electromagnetic scattering problems, this condition is equivalent to assuming the medium is non-magnetic, i.e. $\mu(i)=\mu_{0}$.

\subsection{D media}

In figure 1 we presented an intuitive explanation of the relationship between the actual and warped media. In this section, a more rigorous development of the 1D case is presented. We warp the medium by means of a two-step process. First, divide the existing medium into equal time steps by 'sub-indexing'

$$
\hat{i}_{l+1}=\hat{i}_{l}+\frac{1}{N_{\hat{i}_{l}}} .
$$

Note that the slow layers (given by $N_{i} \neq 1$ ) are subdivided so that all divisions are the same length in time. Second, the new indexing $\hat{i}_{l}$ is used to map the original effective index profile $\{N\}$ onto the indexing defined by $l$ giving the effective index profile of the warped medium $\{\tilde{N}\}(\sim$ denotes a warped medium parameter throughout):

$$
\tilde{N}_{l_{i}}=N_{i} \text {. }
$$

To help illustrate the warping procedure, an example follows using the medium shown in figure 1. Note that the index profile of the warped medium $\tilde{N}_{l}$ does not affect wave speed as did the profile $N_{\hat{i}}$ of the actual medium; the whole point of warping the medium was to obtain an equivalent constant wave speed medium. 
Actual medium:

$$
N_{0}=1, N_{1}=1, N_{2}=2, N_{3}=3, N_{4}=1, \ldots
$$

Sub-indexed medium:

$$
\begin{aligned}
& \hat{i}_{0}=0, \hat{i}_{1}=1, \hat{i}_{2}=1 \frac{1}{2}, \hat{i}_{3}=2, \hat{i}_{4}=2 \frac{1}{3}, \hat{i}_{5}=2 \frac{2}{3}, \hat{i}_{6}=3, \hat{i}_{7}=4 \\
& N_{0}=1, N_{1}=1, N_{1 \frac{1}{2}}=2, N_{2}=2, N_{2 \frac{1}{3}}=3, N_{2 \frac{2}{3}}=3, N_{3}=3, N_{4}=1, \ldots
\end{aligned}
$$

Warped medium:

$$
\tilde{N}_{0}=1, \tilde{N}_{1}=1, \tilde{N}_{2}=2, \tilde{N}_{3}=2, \tilde{N}_{4}=3, \tilde{N}_{5}=3, \tilde{N}_{6}=3, \tilde{N}_{7}=1, \ldots
$$

If the continuous medium has a continuous effective index profile, we note that the warped medium is a piecewise constant approximation over uniform intervals in space and time. Its variable wave speed equivalent, the sub-indexed medium, is a piecewise constant approximation over uniform intervals in time but variable intervals $\{1 / N\}$ in space.

\section{2. $2 D$ media}

In this section, the $1 \mathrm{D}$ warping technique of section 3.1 is extended to the $2 \mathrm{D}$ case. Once again, we assume that the local wave speed is quantized to a rational number, which can be written as before as $\dot{c}_{0} / N(i, j)$ for some integers $c_{0}$ and $N(i, j)$.

Consider two media, both with varying effective index profiles. In the first medium (actual), the effective index variations $N(i, j)$ cause the wave speed in the medium to vary (i.e. $c(i, j)=c_{0} / N(i, j)$ ). In the other medium (warped; denoted by $\sim$ ), the effective index $\tilde{N}(l, m)$ does not affect wave speed (i.e. $c(i, j)=c_{0}$ ). The warped medium does not physically exist; we are merely using it to develop a discrete-time scattering model as will be shown shortly. In order for the warped medium to have the same time sampled wave field as that of the actual medium, the warped medium must be a distorted or warped form of the actual medium. As with the 1D case we warp the actual medium in a two-step process using the medium's effective index profile $N(i, j)$.

Assuming the effective index $\{N\}$ profile to be locally isotropic, the distance that waves travel $\left\{\Delta_{x}, \Delta_{z}\right\}$ during each time step $\Delta_{t} \equiv 1$ is $\Delta_{x}=\Delta_{z}=1 / N(i, j)$ (compare with (3.1)). As in the $1 \mathrm{D}$ case, the first step is to sub-index the grid.

$$
\begin{aligned}
& \hat{i}_{l+1, m}=\hat{i}_{l, m}+\frac{1}{N\left(\hat{i}_{l, m}, \hat{j}_{l, m}\right)} \\
& \hat{j}_{l, m+1}=\hat{j}_{l, m}+\frac{1}{N\left(\hat{i}_{l, m}, \hat{j}_{l, m}\right)} .
\end{aligned}
$$

Note that the slower the region is, the more that it is sub-indexed (i.e. more grid squares are created). In the warped medium $\Delta_{x}=\Delta_{z}=1$ (since $c(i, j)=c_{0}$ ). That is, in the warped medium the waves travel $N(i, j)$ times the distance they do in the actual medium. We find the warped medium $\tilde{N}(l, m)$ by using the appropriate warped grid $(l, m)$ onto which the actual effective index profile $N(i, j)$ is mapped.

$$
\tilde{N}_{l, m}=N\left(\hat{i}_{l, m}, \hat{j}_{l, m}\right)
$$

\section{New variable-mesh finite-difference approximation of the Schrödinger equation}

In this section, we begin with the Schrödinger equation and, using the central difference approximation, find the most general discretization. In the subsequent subsections, 
different temporal and spatial discretization constraints are used to find a constant wave speed equivalent of the variable wave speed medium. These latter discretizations of the Schrödinger equations can be viewed as a variable-mesh finite-difference approximation.

The Schrödinger equation in the time domain for a $2 \mathrm{D}$ variable wave speed medium can be represented in terms of a scattering potential $\{V(x, z)\}$ as follows (see section 2.2 for a derivation)

$$
\nabla^{2} \Psi(x, z, t)-\frac{1}{c^{2}(x, z)} \frac{\partial^{2} \Psi(x, z, t)}{\partial t^{2}}-V(x, z) \Psi(x, z, t)=0 .
$$

$\Psi(x, z, t)$ is the wave field, where $x$ denotes breadth, $z$ denotes depth and $t$ denotes time. $c(x, z)$ is the wave speed and is a function of the effective index $\{N(x, z) \geqslant 1\}$; i.e. $c(x, z)=c_{0} / N(x, z)$, where $c_{0}$ is the least common denominator of the rational slowness at all points. The scattering potential $\{V(x, z)\}$ is a function of the local reflectivities $\left\{r_{x}(x, z), r_{z}(x, z)\right\}$ of the medium which are themselves a first derivative function of the effective index profile $\{N(x, z)\}$ (again see section 2).

\subsection{The general discrete Schrödinger equation}

Using the central difference approximation, we discretize (4.1) to find

$$
\begin{aligned}
& \frac{u_{i, j+\Delta}^{n}+u_{i, j-\Delta}^{n}-2 u_{i, j}^{n}}{\Delta^{2}}+\frac{u_{i+\Delta, j}^{n}+u_{i-\Delta, j}^{n}-2 u_{i, j}^{n}}{\Delta^{2}} \\
& -\frac{N_{i, j}^{2}}{c_{0}^{2}} \frac{u_{i, j}^{n+\Delta_{t}}+u_{i, j}^{n-\Delta_{t}}-2 u_{i, j}^{n}}{\Delta_{t}^{2}}-V_{i, j} u_{i, j}^{n}=0 .
\end{aligned}
$$

To emphasize the discrete 2D nature of (4.2), note that the following change of variables has been made: $\Psi(x, z, t) \rightarrow u_{i, j}^{n}$ (wave field), $t \rightarrow n$ (time), $z \rightarrow i$ (space: depth) and $x \rightarrow j$ (space: breadth). In addition, we denote the discrete space and time increments by the changes $\partial \tau_{x} \rightarrow \Delta, \partial \tau_{z} \rightarrow \Delta$ and $\partial t \rightarrow \Delta_{t}$. We assume $N(x, z)$ is slowly and smoothly varying over $\Delta$, so that (4.2) is a valid approximation of the continuous Schrödinger equation (4.1). Throughout we consider (4.2) as the starting point for our problem, which is therefore explicitly discrete. Define the following discrete quantities (not defined from (2.10), but discretely analogous to (2.10)):

$$
\begin{aligned}
V_{i, j} & =r_{i, j}^{x} r_{i, j-1}^{x}+r_{i, j}^{z} r_{i-1, j}^{z}-\frac{r_{i, j}^{x}-r_{i, j-1}^{x}}{\Delta}-\frac{r_{i, j}^{z}-r_{i-1, j}^{z}}{\Delta} \\
r_{i, j}^{x} & =-\frac{2}{\Delta} \frac{N_{i, j+1}-N_{i, j}}{N_{i, j+1}+N_{i, j}} \\
r_{i, j}^{z} & =-\frac{2}{\Delta} \frac{N_{i+1, j}-N_{i, j}}{N_{i+1, j}+N_{i, j}} .
\end{aligned}
$$

Substituting equations (4.3b) and (4.3c) into (4.3a) yields the 2D scattering potential $V_{i, j}$ :

$$
\begin{array}{r}
V_{i, j}=\frac{4}{\Delta^{2}} \frac{N_{i, j+1} N_{i, j}-2 N_{i, j+1} N_{i, j-1}+N_{i, j} N_{i, j-1}}{N_{i, j}^{2}+N_{i, j+1} N_{i, j}+N_{i, j} N_{i, j-1}+N_{i, j+1} N_{i, j-1}} \\
+\frac{4}{\Delta^{2}} \frac{N_{i+1, j} N_{i, j}-2 N_{i+1, j} N_{i-1, j}+N_{i, j} N_{i-1, j}}{N_{i, j}^{2}+N_{i+1, j} N_{i, j}+N_{i, j} N_{i-1, j}+N_{i+1, j} N_{i-1, j}} .
\end{array}
$$




\subsection{The sub-indexed discrete Schrödinger equation}

Using the sub-indexing concept developed in section $3.2(i \rightarrow \hat{i}, j \rightarrow \hat{j})$, we proceed to provide an alternative discretization of (4.1). By noting that the distance a wave travels during time increment $\Delta_{t}$ depends on the local wave speed $c_{\hat{i}, \hat{j}}$, we have

$$
c_{\hat{i}, \hat{j}} \Delta_{t}=\Delta_{\hat{i}, \hat{j}}
$$

By defining the temporal discretization to be uniformly $\Delta_{t}=\Delta / c_{0}$, the spatiallyvarying discretization is thus given by $\Delta_{\hat{i}, \hat{j}}=\Delta / N_{\hat{i}, \hat{j}}$. In the following, for convenience of notation, we omit the factor $\Delta$ in all indices. We assume that $\Delta$ is sufficiently small that $\Delta_{\hat{i}, \hat{j}}=\Delta / N_{\hat{i}, \hat{j}}$ is also sufficiently small for the variable-discretization finite-difference approximation to be valid. This leads to

$$
\begin{gathered}
{\left[u_{\hat{i}, \hat{j}+1 / N_{\hat{i}, \hat{j}}}^{n}+u_{\hat{i}, \hat{j}-1 / N_{\hat{i}, \hat{j}}}^{n}-2 u_{\hat{i}, \hat{j}}^{n}\right]+\left[u_{\hat{i}+1 / N_{\hat{i}, \hat{j}}, \hat{j}}^{n}+u_{\hat{i}-1 / N_{\hat{i}, \hat{j}}, \hat{j}}^{n}-2 u_{\hat{i}, \hat{j}}^{n}\right]} \\
-\left[u_{\hat{i}, \hat{j}}^{n+1}+u_{\hat{i}, \hat{j}}^{n-1}-2 u_{\hat{i}, \hat{j}}^{n}\right]-V_{\hat{i}, \hat{j}} u_{\hat{i}, \hat{j}}^{n}=0 .
\end{gathered}
$$

The sub-indexed scattering potential $V_{\hat{i}, \hat{j}}$ in (4.6) is given by

$$
\begin{aligned}
V_{\hat{i}, \hat{j}}=4 \frac{N_{\hat{i}, \hat{j}+1 / N_{\hat{i}, \hat{j}}} N_{\hat{i}, \hat{j}}-2 N_{\hat{i}, \hat{j}+1 / N_{\hat{i}, \hat{j}}} N_{\hat{i}, \hat{j}-1 / N_{\hat{i}, \hat{j}}}+N_{\hat{i}, \hat{j}} N_{\hat{i}, \hat{j}-1 / N_{\hat{i}, \hat{j}}}}{N_{\hat{i}, \hat{j}}^{2}+} N_{\hat{i}, \hat{j}+1 / N_{\hat{i}, \hat{j}} N_{\hat{i}, \hat{j}}+N_{\hat{i}, \hat{j}} N_{\hat{i}, \hat{j}-1 / N_{\hat{i}, \hat{j}}}+N_{\hat{i}, \hat{j}+1 / N_{i, \hat{j}}} N_{\hat{i}, \hat{j}-1 / N_{\hat{i}, \hat{j}}}} \\
+4 \frac{N_{\hat{i}+1 / N_{\hat{i}, \hat{j}}, \hat{j}} N_{\hat{i}, \hat{j}}-2 N_{\hat{i}+1 / N_{\hat{i}, \hat{j}}, \hat{j}} N_{\hat{i}-1 / N_{\hat{i}, \hat{j}}, \hat{j}}+N_{\hat{i}, \hat{j}} N_{\hat{i}-1 / N_{\hat{i}, \hat{j}}, \hat{j}}}{N_{\hat{i}, \hat{j}}^{2}+N_{\hat{i}+1 / N_{\hat{i}, \hat{j}}, \hat{j}} N_{\hat{i}, \hat{j}}+N_{\hat{i}, \hat{j}} N_{\hat{i}-1 / N_{\hat{i}, \hat{j}}, \hat{j}}+N_{\hat{i}+1 / N_{\hat{i}, \hat{j}}, \hat{j}} N_{\hat{i}-1 / N_{\hat{i}, \hat{j}}, \hat{j}}} .
\end{aligned}
$$

Recall from section 3 that what we have represented in (4.6) and (4.7) is a discretization on a conformal variable spatial mesh and a uniform temporal mesh. In the following section we warp the spatial indices resulting in a discretization on an equivalent constant wave speed medium that is uniform in time and space.

\subsection{The equivalent constant wave speed discrete Schrödinger equation}

Using the second warping step discussed in section 3.2, we now find the discrete Schrödinger equation that is the constant wave speed equivalent of (4.6). The relationship between the sub-indexed and warped media is

$$
\hat{i} \rightarrow \hat{i} \pm \frac{1}{N_{\hat{i}, \hat{j}}} \Rightarrow l \rightarrow l \pm 1 \quad \hat{j} \rightarrow \hat{j} \pm \frac{1}{N_{\hat{i}, \hat{j}}} \Rightarrow m \rightarrow m \pm 1 .
$$

It is evident from the indexing strategy of (4.8) that the sub-indexed medium is discretized on a uniform temporal mesh and variable spatial mesh, whereas the equivalent constant wave speed medium is discretized on a uniform temporal and a uniform (in the warped coordinates) spatial mesh. The discrete Schrödinger equation for the warped medium is

$$
\begin{array}{r}
{\left[\tilde{u}_{l, m+1}^{n}+\tilde{u}_{l, m-1}^{n}-2 \tilde{u}_{l, m}^{n}\right]+\left[\tilde{u}_{l+1, m}^{n}+\tilde{u}_{l-1, m}^{n}-2 \tilde{u}_{l, m}^{n}\right]} \\
-\left[\tilde{u}_{l, m}^{n+1}+\tilde{u}_{l, m}^{n-1}-2 \tilde{u}_{l, m}^{n}\right]-\tilde{V}_{l, m} \tilde{u}_{l, m}^{n}=0 .
\end{array}
$$

The warped scattering potential $\tilde{V}_{l, m}$ in (4.9) is

$$
\begin{aligned}
& \tilde{V}_{l, m}=4 \frac{\tilde{N}_{l, m+1}}{\tilde{N}_{l, m}-2 \tilde{N}_{l, m+1} \tilde{N}_{l, m-1}+\tilde{N}_{l, m} \tilde{N}_{l, m-1}} \tilde{N}_{l, m}^{2}+\tilde{N}_{l, m+1} \tilde{N}_{l, m}+\tilde{N}_{l, m} \tilde{N}_{l, m-1}+\tilde{N}_{l, m+1} \tilde{N}_{l, m-1} \\
&+4 \frac{\tilde{N}_{l+1, m} \tilde{N}_{l, m}-2 \tilde{N}_{l+1, m} \tilde{N}_{l-1, m}+\tilde{N}_{l, m} \tilde{N}_{l-1, m}}{\tilde{N}_{l, m}^{2}+\tilde{N}_{l+1, m} \tilde{N}_{l, m}+\tilde{N}_{l, m} \tilde{N}_{l-1, m}+\tilde{N}_{l+1, m} \tilde{N}_{l-1, m}} .
\end{aligned}
$$


With (4.9) and (4.10), we now have an equivalent constant wave speed formulation of the discrete Schrödinger equation for a variable wave speed medium.

\subsection{Laterally transformed discrete Schrödinger equation}

In this section we quickly review the Schrödinger equation formulation discussed in [1]. We redefine the discrete 2D Schrödinger equation scattering problem from (4.9) as follows. A wave field $\tilde{u}_{l, m}^{n}$, where $l$ is depth, $m$ is lateral position and $n$ is time, satisfies the $2 \mathrm{D}$ discrete Schrödinger equation

$\tilde{u}_{l+1, m}^{n}+\tilde{u}_{l-1, m}^{n}+\tilde{u}_{l-1, m+1}^{n}+\tilde{u}_{l-1, m-1}^{n}-2 \tilde{u}_{l-1, m}^{n}-\tilde{u}_{l, m}^{n+1}-\tilde{u}_{l, m}^{n-1}=\tilde{V}_{l-1, m} \tilde{u}_{l-1, m}^{n}$.

It is clear that since the indices in (4.11) are actually multiples of $\Delta$, then (4.11) becomes the time domain form of the continuous Schrödinger equation (4.1) as $\Delta \rightarrow 0$. Note that the differences corresponding to $\partial^{2} / \partial x^{2}$ are shifted in depth $z$ from the differences corresponding to $\partial^{2} / \partial z^{2}$ and $\partial^{2} / \partial t^{2}$ as given in (4.9) $\left(\tilde{u}_{l-1, m+1}^{n}+\tilde{u}_{l-1, m-1}^{n}\right.$ instead of $\left.\tilde{u}_{l, m+1}^{n}+\tilde{u}_{l, m-1}^{n}\right)$. This is necessary to obtain the multichannel two-component wave system below.

A discrete-time Fourier transform of (4.1) taking $m$ into $k_{x}$ gives

$$
\begin{aligned}
& u_{l+1, k_{x}}^{n}+u_{l-1, k_{x}}^{n}-u_{l, k_{x}}^{n+1}+u_{l, k_{x}}^{n-1}=\left(2-\mathrm{e}^{\mathrm{i} k_{x}}-\mathrm{e}^{-\mathrm{i} k_{x}}\right) u_{l-1, k_{x}}^{n}+\sum_{m=-\infty}^{\infty} V_{l-1, k_{x}-m}^{n} u_{l-1, m}^{n} \\
& u_{l, k_{x}}^{n}=\sum_{m=-\infty}^{\infty} \tilde{u}_{l, m}^{n} \mathrm{e}^{-\mathrm{i} m k_{x}} ; \quad V_{l, k_{x}}=\frac{1}{2 \pi} \sum_{m=-\infty}^{\infty} \tilde{V}_{l, m} \mathrm{e}^{-\mathrm{i} m k_{x}}
\end{aligned}
$$

Note that $2-\mathrm{e}^{\mathrm{i} k_{x}}-\mathrm{e}^{-\mathrm{i} k_{x}}=2\left(1-\cos k_{x}\right) \approx k_{x}^{2}$ as $k_{x} \rightarrow 0$. This also shows how the discrete 2D Schrödinger equation reduces to the continuous 2D Schrödinger equation in the limit as $\Delta \rightarrow 0$. Note that the ill-conditioned lateral derivative $\partial^{2} / \partial x^{2}$, which makes downward continuation ill-posed, is regularized to the smoothed wavenumber response $2\left(1-\cos k_{x}\right)$. The boundary conditions for (4.12) are

$$
u_{l, k_{x} ; k^{\prime}}^{n}= \begin{cases}\delta(n-l) \delta\left(k_{x}-k_{x}^{\prime}\right)+R\left(n+l, k_{x} ; k_{x}^{\prime}\right) & \text { if } l \leqslant 0 \\ T\left(n-l, k_{x} ; k_{x}^{\prime}\right) & \text { if } l \rightarrow \infty\end{cases}
$$

where $R\left(n, k_{x} ; k_{x}^{\prime}\right)$ is the reflection response and $T\left(n, k_{x} ; k_{x}^{\prime}\right)$ is the transmission response to an impulsive plane wave that excites the lateral wavenumber $k_{x}^{\prime}$ only.

\section{The forward scattering problem}

In the next two sections, we use (4.12) as a basis for the forward and inverse scattering problems which are solved using layer stripping algorithms as discussed in [1]. The discrete 2D forward scattering problem is to reconstruct $R\left(n, k_{x} ; k_{x}^{\prime}\right)$ from $V_{l, k_{x}}$. We discretize $k_{x}$ into integer multiples of $\Delta$. Equation (4.12) can then be rewritten as

$$
\boldsymbol{u}_{l+1}^{n}+\boldsymbol{u}_{l-1}^{n}-\boldsymbol{u}_{l}^{n+1}-\boldsymbol{u}_{l}^{n-1}=\boldsymbol{V}_{l-1} \boldsymbol{u}_{l-1}^{n}
$$

where $\boldsymbol{u}_{l}^{n}$ is a matrix whose $(m, o)$ th element is $u_{l, m ; o}^{n}$ and $\boldsymbol{V}_{l}$ is a Toeplitz-plus-diagonal matrix with $(m, o)$ th element $(\delta(m-o)$ is now a Kronecker delta)

$$
\boldsymbol{V}_{l, m ; o}=2(1-\cos (m \Delta)) \delta(m-o)+V(l,(m-o) \Delta) \Delta .
$$


The indices in (5.2) run from $-M$ to $M$, where $M$ is an arbitrarily large integer, and $\boldsymbol{V}$ is Hermitian, since $\tilde{V}_{l, m}$ is real. Given the matrix potential $\boldsymbol{V}_{l}(5.2)$, define the matrix reflectivity $\boldsymbol{r}_{l}$ recursively from the transform [9]

$$
\begin{aligned}
& \boldsymbol{r}_{l}=\boldsymbol{T}_{l}^{-1}\left(I-\boldsymbol{V}_{l}\right) \boldsymbol{T}_{l}\left(I-\boldsymbol{r}_{l-1}\right)^{-1}-I \\
& \boldsymbol{T}_{l}=\prod_{p=1}^{l-1}\left(I-\boldsymbol{r}_{p}^{2}\right)^{1 / 2} \\
& \boldsymbol{u}_{l}^{n}=\boldsymbol{T}_{l}\left(I-\boldsymbol{r}_{l-1}\right)^{-1}\left(\boldsymbol{D}_{l}^{n}+\boldsymbol{U}_{l}^{n}\right) .
\end{aligned}
$$

Then the matrix discrete Schrödinger equation (5.1) is equivalent to the discrete multichannel two-component wave system [9]

$$
\left[\begin{array}{c}
\boldsymbol{D}_{l+1}^{n} \\
\boldsymbol{U}_{l+1}^{n}
\end{array}\right]=\left[\begin{array}{cc}
\left(I-\boldsymbol{r}_{l}^{2}\right)^{-\frac{1}{2}} & \mathbf{0} \\
\mathbf{0} & \left(I-\boldsymbol{r}_{l}^{2}\right)^{-\frac{1}{2}}
\end{array}\right]\left[\begin{array}{cc}
I & -\boldsymbol{r}_{l} \\
-\boldsymbol{r}_{l} & I
\end{array}\right]\left[\begin{array}{c}
\boldsymbol{D}_{l}^{n-1} \\
\boldsymbol{U}_{l}^{n+1}
\end{array}\right] .
$$

For each of the matrices (the bold quantities), different columns correspond to different experiments, while different rows correspond to different channels for a given experiment.

Now suppose that the top interface is a free surface (perfect reflector), and that the probing impulse in (4.13) is introduced just below this surface; i.e. $I+\boldsymbol{R}(n)=\boldsymbol{D}_{0}^{n}$ and $\boldsymbol{R}(n)=\boldsymbol{U}_{0}^{n}$. The forward scattering problem is to find the response $\boldsymbol{R}$ from the reflectivity $\boldsymbol{r}$ and is solved using (5.4) and the multichannel form of the Schur algorithm run backwards. The multichannel Schur algorithm should be expected to work well, since it solves exactly an explicitly discrete 2D scattering problem. The discrete matrix two-component system (5.4) is lossless and well behaved numerically as long as the maximum singular value of $\boldsymbol{r}$ is less than unity.

\section{The inverse scattering problem}

The discrete 2D inverse scattering problem is to reconstruct $V\left(i, k_{x}\right)$ from $R\left(n, k_{x} ; k_{x}^{\prime}\right)$. The 2D discrete inverse scattering problem defined by (4.12) and (4.13), or equivalently (5.4), can be solved very easily using the multichannel form [9] of the Schur algorithm when incorporating the factor $\left(I-\boldsymbol{r}(i)^{2}\right)^{-1 / 2}$, which produces additional coupling between channels.

\subsection{Reflectivity from impulse reflection response}

The Schur algorithm computes the reflection coefficients associated with the block-Toeplitz system of equations [8]:

$$
\begin{aligned}
& {\left[\begin{array}{cccc}
I & \boldsymbol{R}(1) & \cdots & \boldsymbol{R}(n) \\
\boldsymbol{R}(1) & I & \cdots & \boldsymbol{R}(n-1) \\
\vdots & \vdots & \ddots & \vdots \\
\boldsymbol{R}(n) & \boldsymbol{R}(n-1) & \cdots & I
\end{array}\right]\left[\begin{array}{c}
\boldsymbol{F}_{n}^{0}-\boldsymbol{G}_{n}^{n} \\
\vdots \\
\boldsymbol{F}_{n}^{n}-\boldsymbol{G}_{n}^{0}
\end{array}\right]=\left[\begin{array}{c}
\mathbf{0} \\
\mathbf{0} \\
\vdots \\
\boldsymbol{T}(n)
\end{array}\right]} \\
& \boldsymbol{R ( n ) _ { ( m , o ) } = R ( n , m \Delta ; o \Delta ) .}
\end{aligned}
$$

Note that the $\boldsymbol{R}(n)$ are themselves matrices defined from the reflection response $R\left(n, k_{x} ; k_{x}^{\prime}\right)$ defined in (4.13). $\quad \boldsymbol{F}_{n}^{i}$ and $\boldsymbol{G}_{n}^{i}$ are elements of the matrix Green's function of (5.4). The matrix reflectivity $\boldsymbol{r}_{n}$ can be recovered using $\boldsymbol{r}_{n}=\boldsymbol{G}_{n}^{0}-\boldsymbol{F}_{n}^{n}$. Equation (6.1) can thus be viewed as a discrete analogue of an integral equation, and the Schur algorithm as a fast algorithm alternative to solving this discrete integral equation analogue. 


\subsection{Warped scattering potential from reflectivity}

Reformulating the transform given in (5.3) enables us to directly calculate the laterally transformed scattering potential corresponding to the equivalent warped medium as

$$
\begin{aligned}
\boldsymbol{V}_{l} & =I-\boldsymbol{T}_{l}\left(I+\boldsymbol{r}_{l}\right)\left(I-\boldsymbol{r}_{l-1}\right) \boldsymbol{T}_{l}^{-1} \\
\boldsymbol{T}_{l} & =\prod_{p=1}^{l-1}\left(I-\boldsymbol{r}_{p}^{2}\right)^{1 / 2} .
\end{aligned}
$$

\subsection{Warped effective index profile from warped scattering potential}

From equation (4.9) we clearly see that the scattering potential $\tilde{V}_{l, m}$ of the warped medium can be found when the warped effective index profile $\tilde{N}_{l, m}$ is known. For the inverse problem we wish to find a recursive relationship in which to find $\tilde{N}_{l, m}$ from $\tilde{V}_{l, m}$. We assume that the probing layer is known and that we reconstruct the profile layer by layer. That is, in addition to knowing $\tilde{V}_{l, m}$, we know $\tilde{N}_{l^{\prime}, m^{\prime}}, \forall l^{\prime} \leqslant l$ and $\forall m^{\prime}$. For ease of notation, let

$$
\tilde{L}_{l, m}=\frac{\tilde{N}_{l, m+1} \tilde{N}_{l, m}-2 \tilde{N}_{l, m+1} \tilde{N}_{l, m-1}+\tilde{N}_{l, m} \tilde{N}_{l, m-1}}{\tilde{N}_{l, m}^{2}+\tilde{N}_{l, m+1} \tilde{N}_{l, m}+\tilde{N}_{l, m+1} \tilde{N}_{l, m-1}+\tilde{N}_{l, m} \tilde{N}_{l, m-1}} .
$$

Since $\tilde{V}_{l, m}, \tilde{L}_{l, m}$ and the appropriate $\tilde{N}_{l^{\prime}, m^{\prime}}$, are all known, we have the simple recursion

$$
\tilde{N}_{l+1, m}=\frac{\left(\left(\tilde{V}_{l, m} / 4\right)-\tilde{L}_{l, m}\right)\left(\tilde{N}_{l, m}^{2}-\tilde{N}_{l, m} \tilde{N}_{l-1, m}\right)-\tilde{N}_{l, m} \tilde{N}_{l-1, m}}{\tilde{N}_{l, m}-2 \tilde{N}_{l-1, m}-\left(\left(\tilde{V}_{l, m} / 4\right)-\tilde{L}_{l, m}\right)\left(\tilde{N}_{l, m}+\tilde{N}_{l-1, m}\right)} .
$$

\subsection{Variable wave speed effective index profile from warped profile}

The final step of the inverse problem is to determine the actual medium from the reconstructed warped medium. That is, one must determine the actual index profile $\left\{N_{\hat{i}}\right\}$ from the warped medium's index profile $\left\{\tilde{N}_{l}\right\}$. We call this process dewarping of the reconstructed medium, since it is essentially performing the methodology of section 3 backwards.

As with the warping of the actual medium, dewarping of the equivalent is a two-step process. First, we form the sub-indexed grid $(\hat{i}, \hat{j})$ on which we map the warped index profile $\tilde{N}_{l, m}$ :

$$
\begin{gathered}
\hat{i}_{l+1, m}=\hat{i}_{l, m}+\frac{1}{\tilde{N}_{l, m}} \\
\hat{j}_{l, m+1}=\hat{j}_{l, m}+\frac{1}{\tilde{N}_{l, m}} \\
N\left(\hat{i}_{l, m}, \hat{j}_{l, m}\right)=\tilde{N}_{l, m} .
\end{gathered}
$$

From (6.5), note that the slower the region is, the more the grid is compressed. Note also that there is a one-to-one relationship between the warped $\left(\tilde{N}_{l, m}\right)$ and sub-indexed $(N(\hat{i}, \hat{j}))$ effective index profile as given by $(6.5 c)$. Finally, the original effective index profile $N(i, j)$ can be found by interpolating $N(\hat{i}, \hat{j})$ onto a uniform spatial mesh $(i, j)$. 

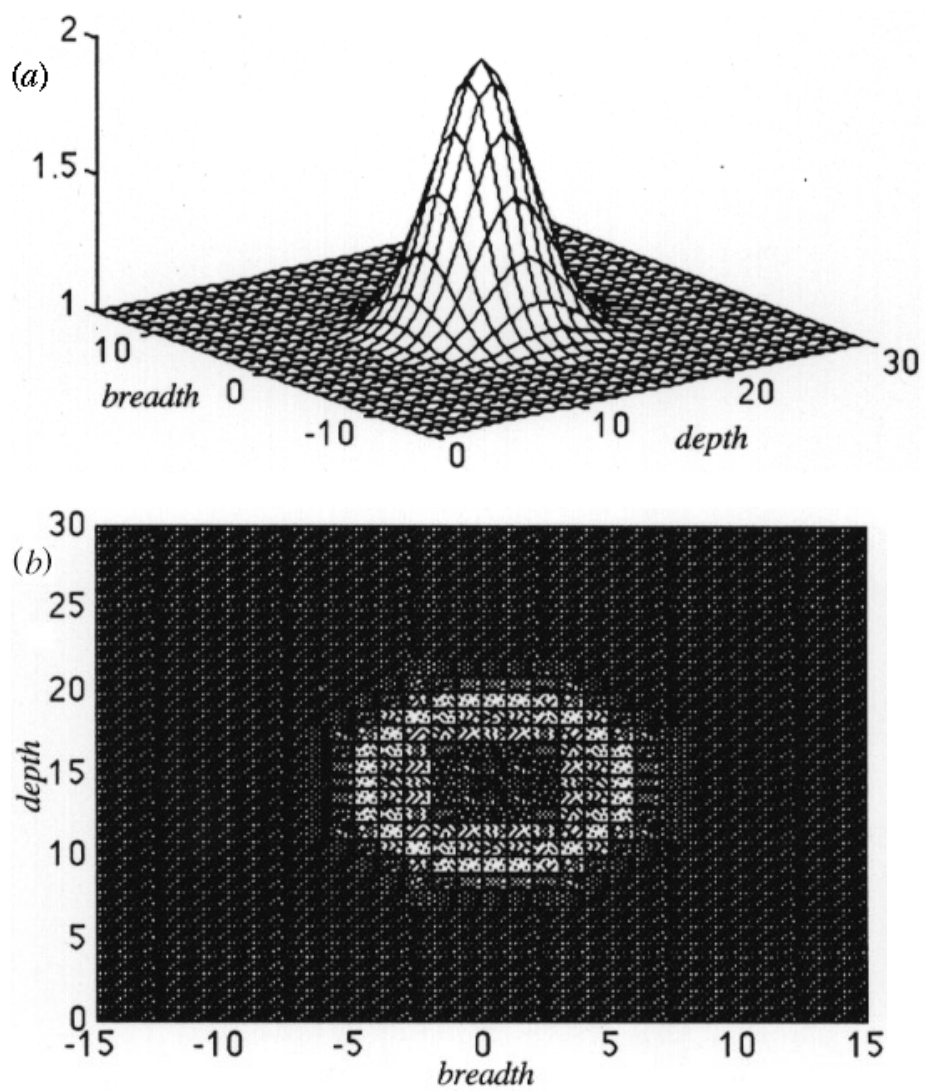

Figure 2. Actual medium. (a) Effective index profile; (b) uniform spatial discretization.

\section{Numerical example}

In this section we present a numerical example to illustrate the methodology presented in this paper. We begin with a variable wave speed medium on a uniform spatial grid, then determine the corresponding sub-indexed medium, which we then warp to find our equivalent constant wave speed medium. The scattering potential of the constant wave speed medium is found from the warped index profile. The impulse reflection response of the medium can be found from this warped scattering potential by running the Schur layer stripping algorithm backwards. The inverse scattering problem can be solved by running the Schur layer stripping algorithm, and then dewarping as discussed in section 6 . The effects of noise in the data on reconstruction and data feasibility criteria were addressed in [1].

\subsection{Original medium}

We consider a 2D variable wave speed medium in which there exists a graded effective index inclusion having a Gaussian profile as shown figure 2(a). Numerical methods, such as FDTD and TLM, model the medium using a uniform spatial mesh as shown in figure 2(b). Note that this amounts to a low wave speed inclusion in the medium. 


\subsection{Sub-indexed medium}

Using the methodology of section 3, we sub-index the actual medium (figure 2) creating the sub-indexed contour shown in figure 3. Note that the sub-indexed contour (figure 3 ) has approximately the same shape as the original contour (figure $2(b)$ ) but with more grid points in regions of higher effective index. Increasing the number of grid points (i.e. sub-indexing at a higher rate) would improve the contour approximation. Note also that all squares in figure 3 represent equal time increments.

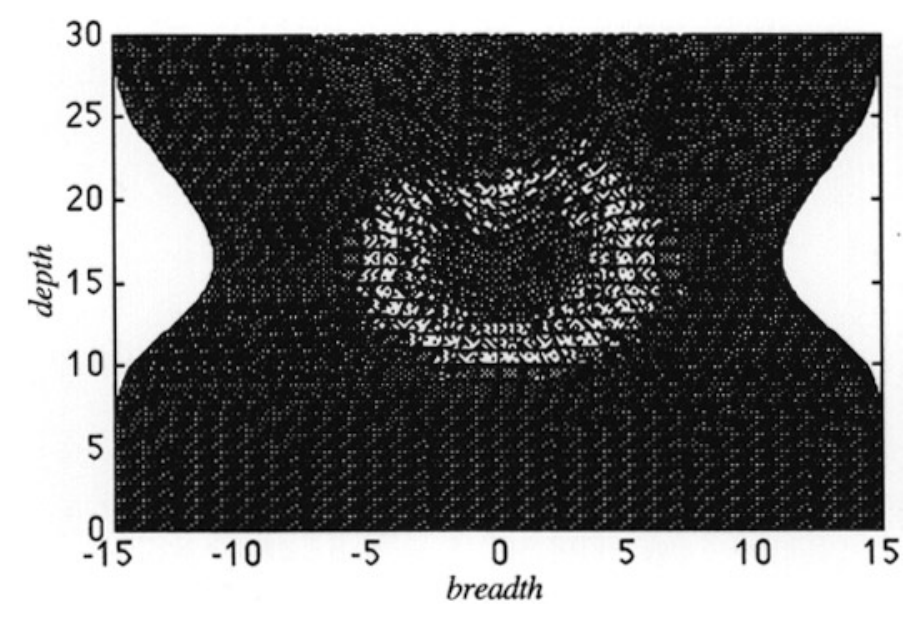

Figure 3. Variable spatial mesh of sub-indexed medium.

\subsection{Equivalent constant wave speed medium}

By warping (i.e. re-indexing), the sub-indexed medium (figure 3), we have our desired equivalent constant wave speed medium (figure 4) which is discretized on a uniform spatial mesh corresponding to a uniform temporal mesh. Note that the contour of the warped medium no longer approximates that of the actual medium (figure 2(a)). The warped medium is distorted both in depth (vertical axis) and breadth (horizontal axis) as is prescribed by the methodology of section 3 and, in particular, the 2D warping equations (3.3) and (3.4).

\subsection{The forward scattering problem}

The reflection response $R_{n, m}$ is found from $\tilde{V}_{l, m}$ by first finding the matrix reflectivity $\boldsymbol{r}$ using (5.3) and then running the multichannel Schur algorithm backwards. Recall that $R_{n, m}$ is the free-surface impulse reflection response as measured at the surface boundary of the medium (figure 5).

To better appreciate the effects of wave speed variation, we show in figure 6 snap shots of the probing wave and scattered field as they propagate through the medium. Note that the wave travels further in the faster sections of the medium as time progresses and the scattering that occurs as the wave speed changes. 


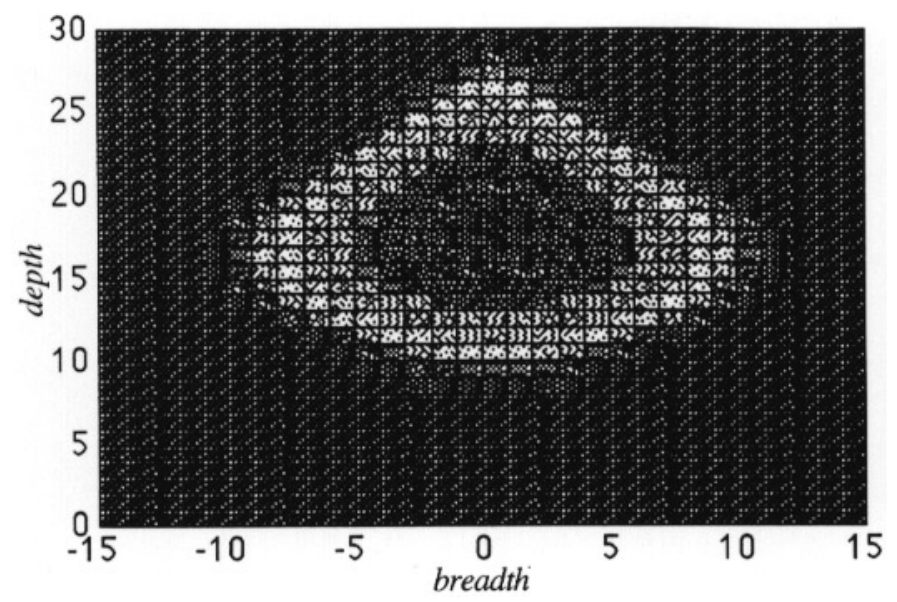

Figure 4. Uniform temporal and spatial mesh of warped medium.

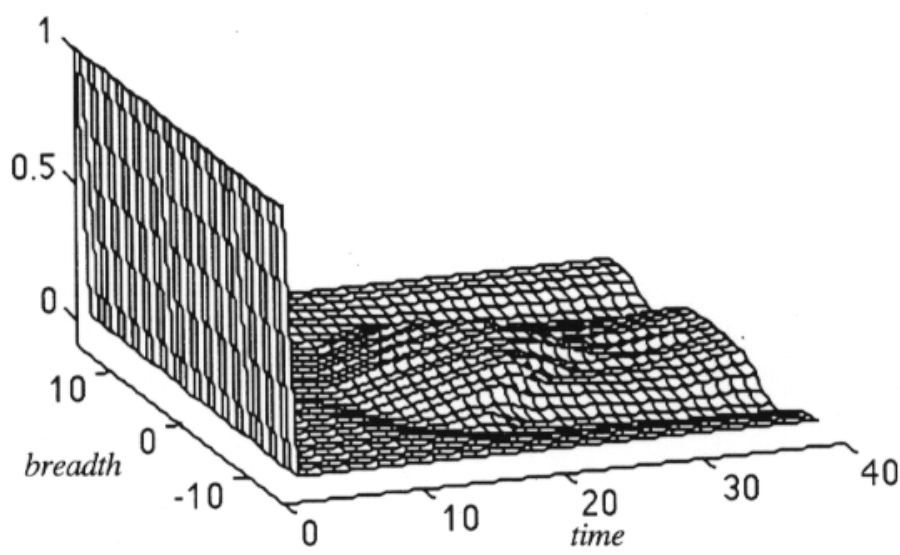

Figure 5. Reflection response for medium.

\section{The inverse scattering problem}

The inverse scattering problem is to reconstruct the wave speed profile from observations of the scattered field at the surface $z=0$ (shown in figure 5). To do this we proceed as follows.

1. Run the Schur layer stripping algorithm on the observed reflected scattered field data. This incorrectly assumes uniform wave speed, but generates the correct values of the scattered field and reflectivity function $\{\tilde{r}(l, m)\}$, but at the wrong locations.

2. Compute the warped medium index profile $\{\tilde{N}(l, m)\}$ from the potential function $\{\tilde{V}(l, m)\}(6.3)$ and (6.4). The potential function is computed directly from the reflectivity function (6.2).

3. Dewarp the medium using (6.5). This yields the sub-indexed medium $\{N(\tilde{i}, \tilde{j})\}$ shown in figure 3.

4. Interpolate the sub-indexed medium onto a uniform spatial grid. This yields the effective index on a uniform spatial grid $\{N(i, j)\}$, which in turn interpolates uniformly to $N(x, z)$. 

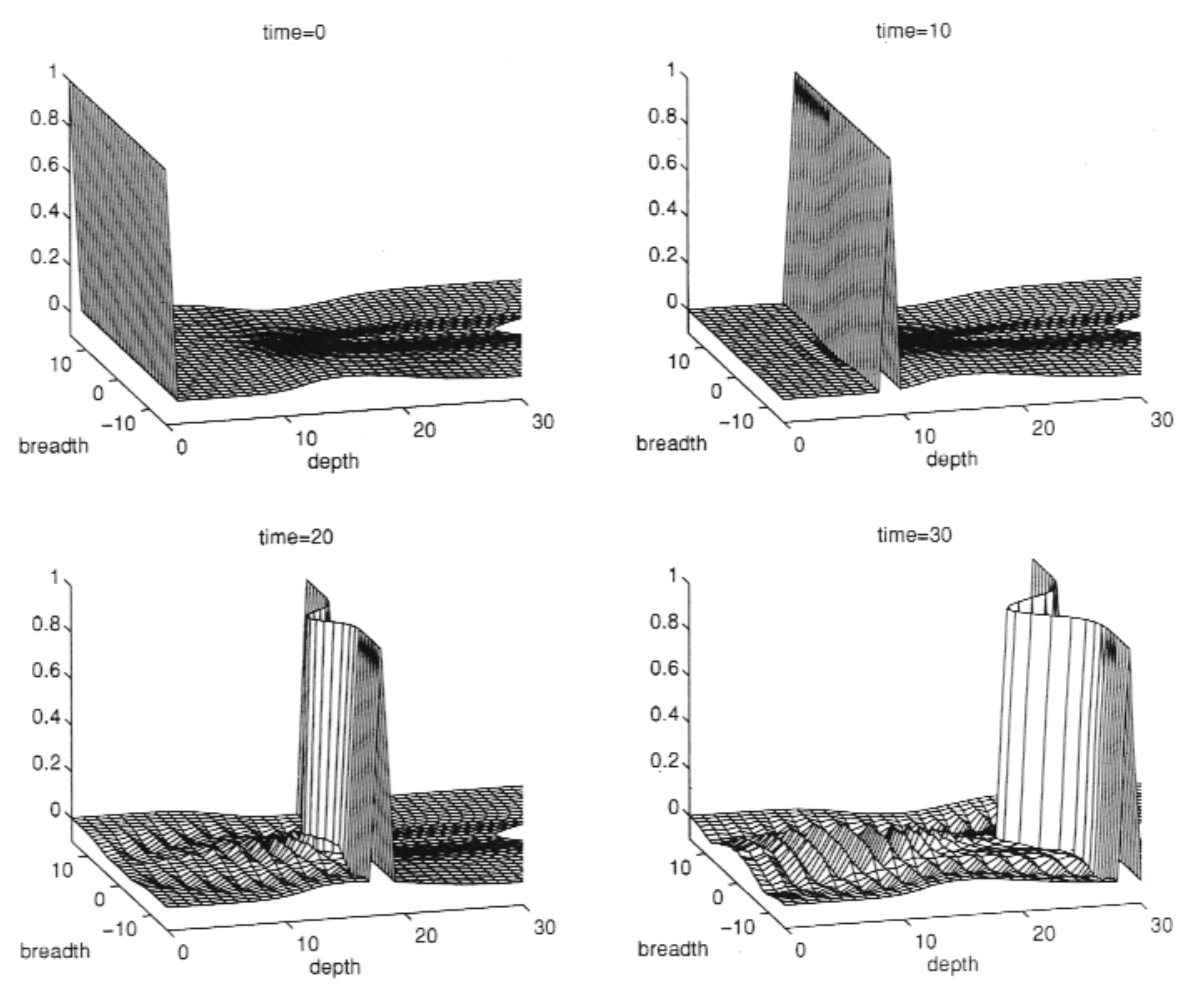

Figure 6. Propagating and scattering waves.

5. Compute the wave speed profile from the effective index profile using $c(x, z)=$ $c_{0} / N(x, z)$.

Following this procedure correctly reconstructed the wave speed profile corresponding to the effective index profile shown in figure 2. The effects of noise on 2D Schur layer stripping algorithms were discussed in detail in [1]. In summary, as long as the data are feasible, a stable but noisy reconstruction will result. In [1] we propose a procedure for correcting noisy and unfeasible data to feasible data. Since the point of this paper is that the variable wave speed problem can be recast as the problem considered in [1], there is no need to repeat the results of [1] for noisy data here.

\section{Conclusion}

In this paper we have shown that the Schrödinger equation for 2D media with variable wave speed may be discretized in one of two equivalent manners: (1) using a fixed square temporal lattice and varying the spatial discretization due to the varying wave speed or (2) fixing the temporal discretization and assuming a constant wave speed, and then warping the medium so that the spatial discretization is also a uniform square lattice. The latter's scattered field is equivalent to the former's scattered field in time but occurs at different places spatially. This formulation can be viewed as a variable-mesh finite-difference approximation which allows for the use of fast algorithms to solve the forward and inverse problems where the scattering is due to the effective index profile of the warped medium. The wave speed 
profile of the warped medium is then used to dewarp the medium to find the desired actual medium.

Future topics of research include applying these formulations to the problem of inclusions having rough interfaces. This application is non-trivial as in this paper we have assumed our inclusion to be smoothly varying and thus the warping of the medium also occurred smoothly. By definition, a rough interface does not have a smooth effective index transition. A possible solution to this problem is to divide the medium into regions according to ranges of effective index and apply the discussed methodology to each region.

\section{References}

[1] Yagle A E and Frolik J L 1996 On the feasibility of impulse reflection response data for the two-dimensional inverse scattering problem IEEE Trans. Ant. Prop. to appear

[2] Frolik J L and Yagle A E 1996 Forward and inverse scattering for discrete layered lossy and absorbing media IEEE Trans. Circuits Syst. B to appear

[3] Taflove A and Umashankar K R 1990 The finite-difference time-domain method for numerical modeling of electromagnetic wave interactions with arbitrary structures PIER-2: Finite Element and Finite Difference Methods in Electromagnetic Scattering ed M A Morgan (New York: Elsevier) ch 8

[4] Smith G D 1985 Numerical Solution of Partial Differential Equations: Finite Difference Methods 3rd edn (Oxford: Oxford University Press)

[5] Hopcraft K I and Smith P R 1992 An Introduction to Electromagnetic Inverse Scattering (Deventer: Kluwer)

[6] Hoefer W J R 1989 The transmission line matrix (TLM) method Numerical Techniques for Microwaves and Millimeter-Wave Passive Structures ed T Itoh (New York: Wiley) ch 8

[7] Yagle A E 1989 One-dimensional inverse scattering problems: an asymmetric two-component wave system framework Inverse Problems 5 641-66

[8] Bruckstein A M and Kailath T 1987 Inverse scattering for discrete transmission line models SIAM Rev. 29 $359-89$

[9] Yagle A E 1989 Discrete matrix Schrödinger equation equivalents of discrete two-component matrix wave systems Inverse Problems 5 425-36 